\title{
Présence de pseudokystes gélatineux en contexte de cryptococcose du système nerveux central
}

\author{
Joy Zhuo Ding MD et Carlos Torres MD
}

Citation : CMAJ 2021 April 12;193:E522. doi : 10.1503/cmaj.201547-f

Voir la version anglaise de l'article ici : www.cmaj.ca/lookup/doi/10.1503/cmaj.201547

$\mathbf{U}$ n homme de 44 ans de la Colombie-Britannique a été hospitalisé après avoir présenté depuis 2 semaines des maux de tête, des vomissements ainsi que des douleurs au cou et au dos dont l'intensité augmentait. Le patient était immunodéprimé, car il utilisait de l'ocrélizumab, de l'azathioprine et de la prednisone pour traiter la sclérose en plaques (SP) et le purpura thrombopénique immunologique. Il ne présentait pas de raideur à la nuque ni de fièvre et son examen clinique demeurait inchangé en lien avec l'état de base de ses déficits neurologiques stables associés à sa sclérose en plaques. L'examen de son cerveau au moyen de l'imagerie par résonance magnétique (IRM) avec agent de contraste au gadolinium a montré la présence de minuscules kystes dans les noyaux gris centraux qui n'étaient pas présents sur les images d'IRM réalisées 4 semaines plus tôt (figure $1 \mathrm{~A}$; annexe 1, figure supplémentaire, accessible en anglais au www. cmaj.ca/lookup/doi/10.1503/cmaj.201547/tab-related-content).

Nous avons retardé une ponction lombaire en raison d'un traitement anticoagulant. Une biopsie d'une masse croissante dans le lobe inférieur droit du poumon a relevé la présence de Cryptococcus gattii. Une ponction lombaire a par la suite démontré des résultats d'analyse du liquide céphalorachidien (LCR) normaux (numération leucocytaire de 2 [plage normale $0-5$ ] $\times 10^{6} / \mathrm{L}$, numération des globules rouges de 4 [plage normale $<1$ ] $\times 10^{6} / \mathrm{L}$, glycémie de 3,6 [plage normale de 2,2-3,9] mmol/L et taux de protéine de 0,48 [plage normale de $0,12-0,6$ ] g/L), mais une pression d'ouverture excédant $55 \mathrm{~cm} \mathrm{H}_{2} \mathrm{O}$. Le liquide céphalorachidien s'est révélé positif à la présence de l'antigène cryptococcique. Malgré un traitement approprié à l'amphotéricine $B$ et à la flucytosine, de même que des ponctions lombaires quotidiennes, un examen
d'IRM cérébral réalisé 2 semaines après de début de l'hospitalisation a montré un accroissement substantiel de la taille et du nombre des pseudokystes gélatineux (figure 1B).

Quatre mois plus tard, le patient a obtenu son congé de l'hôpital et a poursuivi son traitement au fluconazole; cependant, il présentait une perte persistante d'acuité visuelle profonde.

Le Cryptococcus gattii, un champignon qui se trouve dans les arbres et le sol, est endémique en Colombie-Britannique et infecte les êtres humains par l'inhalation de ses spores. L'île de Vancouver présente l'un des taux d'incidence annuels les plus élevés, variant entre 0,1 et 2,2 par 100000 habitants entre 2003 et $2018^{1}$. L'âge avancé est un facteur de risque de l'infection et les patients qui sont immunocompétents sont fréquemment infectés. 
Les résultats obtenus en employant l'imagerie par résonance magnétique dans le contexte de la méningo-encéphalite cryptococcique comprennent des cryptococcomes parenchymateux, des pseudokystes gélatineux, une augmentation leptoméningée et une hydrocéphalie communicante ${ }^{2}$. On observe des pseudokystes gélatineux dans presque $50 \%$ des infections à C. gattii associées au VIH; cependant, sa prévalence chez les patients immunocompétents demeure inconnue ${ }^{3}$. Ces pseudokystes se forment sur une période de plusieurs semaines, mais il peut être difficile de les déceler initialement, particulièrement chez les patients présentant des anomalies préexistantes sur leurs images d'IRM.

Les médecins devraient envisager une infection du système nerveux central à C. gattii chez les patients immunocompétents et immunovulnérables présentant des maux de tête, une cervicalgie et des lésions pulmonaires, et qui ont visité l'île de Vancouver. L'aspect des pseudokystes gélatineux ou d'autres résultats typiques de l'examen d'IRM sont utiles s'ils sont présents. La composition du liquide céphalorachidien peut être normale; le dépistage de l'antigène cryptococcique et la culture du LCR sont requis pour confirmer le diagnostic.

\section{Références}

1. Reportable Diseases Data Dashboard. Vancouver: BC Centre for Disease Control. Accessible ici : www.bccdc.ca/health-professionals/data-reports/reportable-diseases -data-dashboard?Disease=Cryptococcus\%20gattii (consulté le 20 sept. 2020).

2. Duarte SB, Oshima MM, do Amaral Mesquita JV, et al. Magnetic resonance imaging findings in central nervous system cryptococcosis: comparison between immunocompetent and immunocompromised patients. Radiol Bras 2017;50:359-65.

3. Chen SC-A, Meyer W, Sorrell TC. Cryptococcus gattii infections. Clin Microbiol Rev 2014;27:980-1024.

\section{Intérêts concurrents : Aucun déclaré.}

Cet article a été révisé par des pairs.

Les auteurs ont obtenu le consentement du patient.

Affiliations : Service de neurologie (Ding), Hôpital régional Penticton, Penticton, C.-B.; Département de radiologie (Torres), Université d'Ottawa; Service d'imagerie médicale (Torres), Hôpital d'Ottawa, Ottawa, Ont.

Propriété intellectuelle du contenu : Il s'agit d'un article en libre accès distribué conformément aux modalités de la licence Creative Commons Attribution (CC BY-NC-ND 4.0), qui permet I'utilisation, la diffusion et la reproduction de tout médium à la condition que la publication originale soit adéquatement citée, que l'utilisation se fasse à des fins non commerciales (c.-à-d., recherche ou éducation) et qu'aucune modification ni adaptation n'y soit apportée. Voir : https://creativecommons.org/licenses/ by-nc-nd/4.0/deed.fr.

Correspondance : Joy Ding, joy.ding@medportal.ca

Les images cliniques sont choisies pour leur caractère particulièrement intéressant, classique ou impressionnant. Toute soumission d'image de haute résolution claire et bien identifiée doit être accompagnée d'une légende aux fins de publication. On demande aussi une brève explication (300 mots maximum) de la portée éducative des images, et des références minimales. Le consentement écrit du patient au regard de la publication doit être obtenu avant la soumission. 